\title{
INFECC̣ÃO PULMONAR TRIPLA EM PACIENTE GRAVEMENTE IMUNOCOMPROMETIDO POR AIDS: RELATO DE CASO*
}

\author{
Rafael Artigas Faucz ${ }^{1}$, Marianne Siquara de Quadros ${ }^{2}$, Celso Alessandro Andrade ${ }^{3}$, Flávio \\ Trentin Troncoso ${ }^{3}$, Nelson Ribeiro Filho ${ }^{4}$, Juvencio Duailibe Furtado ${ }^{5}$, Ricardo Pires de Souza
}

Resumo Os autores relatam um caso de paciente do sexo masculino, 38 anos de idade, motorista, soropositivo para HIV há oito anos, sem acompanhamento, com quadro de tosse produtiva com secreção acinzentada e episódios intermitentes de dispnéia há 15 dias. Informava dois episódios pregressos de tuberculose pulmonar (1983 e 2001) tratados. A radiografia de tórax evidenciou áreas de hipotransparência nodular e broncogramas aéreos bilateralmente. A tomografia computadorizada de tórax evidenciou vários achados inespecíficos, dentre eles áreas esparsas de consolidação, cavitação, bronquiectasia, opacidade em vidro fosco, espessamento intersticial e broncogramas aéreos. A lavagem broncoalveolar evidenciou numerosas hifas com raros septos bifurcados sugestivos de Aspergillus sp. e a cultura foi positiva para Nocardia sp. e Mycobacterium tuberculosis. Foi instituída terapia com anfotericina $B$, sulfametoxazol-trimetoprim e anti-retrovirais. Após 20 dias, recebeu alta sem queixas pulmonares. Decorridos 15 dias, retornou com diarréia, febre, disfagia e emagrecimento importante. Foi a óbito após cinco dias, por sepse estafilocócica.

Unitermos: SIDA; Tuberculose; Aspergilose; Nocardiose; Radiologia.

Abstract Triple pulmonary infection in a severely immunocompromised AIDS patient: a case report.

The authors report a case of a 38-year-old male patient who works as a driver, HIV positive for eight years and with no follow up since diagnosis presenting with productive cough with grayish secretion and intermittent attacks of dyspnea two weeks before hospital admission. He also reported two previous episodes of treated pulmonary tuberculosis (in 1983 and 2001). Chest X-ray showed nodular hypolucent areas and air bronchograms bilaterally. Computed tomography of the chest revealed many unspecific findings, including scattered areas of consolidation, cavitations, bronchial dilatation, ground glass opacities, interstitial thickening and air bronchograms. The bronchoalveolar lavage showed a number of hyphaes with rare bifurcated septa suggestive of Aspergillus sp. Cultures of Nocardia sp. and Mycobacterium tuberculosis were positive. Therefore, treatment with amphotericin B, trimethoprim-sulfamethoxazole and antiretroviral was started. The patient was discharged twenty days later without pulmonary symptoms. Fifteen days later, the patient returned to the hospital with diarrhea, fever, dysphagia and significant weight loss. The patient died five days later due to staphylococci sepsis.

Key words: AIDS; Tuberculosis; Aspergillosis; Nocardiosis; Radiology.

\section{INTRODUÇÃO}

A infecção pulmonar em pacientes imunossuprimidos é situação clínica bastante comum nos ambulatórios e enfermarias dos

* Trabalho realizado nos Serviços de Radiologia e Diagnóstico por Imagem e de Infectologia do Hospital Heliópolis, São Paulo, SP.

1. Médico Estagiário do Serviço de Radiologia e Diagnóstico por Imagem do Hospital Heliópolis.

2. Médica Residente do Serviço de Radiologia e Diagnóstico por Imagem do Hospital Heliópolis.

3. Médicos Residentes do Serviço de Infectologia do Hospital Heliópolis.

4. Médico Assistente do Serviço de Infectologia do Hospital Heliópolis.

5. Médico Chefe do Serviço de Infectologia do Hospital Heliópolis.

6. Médico Chefe do Serviço de Radiologia e Diagnóstico por Imagem do Hospital Heliópolis.

Endereço para correspondência: Dr. Rafael Artigas Faucz. Rua Doutor Diogo de Faria, 1201, ap. 87, Vila Mariana. São Paulo, SP, 04037-004. E-mail: rafaucz@terra.com.br

Recebido para publicação em 4/3/2005. Aceito, após revisão, em 23/3/2005. infectologistas. Porém, é extremamente raro quando esta infecção é causada por três microorganismos distintos.

O objetivo deste trabalho é relatar um caso de paciente imunodeprimido por síndrome da imunodeficiência adquirida (SIDA) com tripla infecção pulmonar (tuberculose, aspergilose e nocardiose), demonstrar os aspectos de imagem (radiografia simples e tomografia computadorizada [TC] do tórax) e as características anatomopatológicas de cada um desses microorganismos.

\section{RELATO DO CASO}

Paciente de 38 anos de idade, motorista, procedente de São Paulo, soropositivo para o vírus da imunodeficiência humana (HIV) há oito anos, sem acompanhamento médico, queixava-se de tosse produtiva com secreção acinzentada e episódios intermitentes de dispnéia há 15 dias. Informava dois episódios prévios de tuberculose pulmonar (1983 e 2001) tratadas.

A radiografia de tórax mostrou áreas de hipotransparência nodular e broncogramas aéreos bilateralmente (Figura 1). A TC de tórax evidenciou áreas de consolidação, cavitação, bronquiectasia, opacidade em vidro fosco, espessamento intersticial e broncogramas aéreos (Figura 2).

O exame de bacterioscopia do escarro foi negativo para bacilos álcool-ácido resistentes (BAAR) e fungos. Foi instituída antibioticoterapia empírica para pneumonia bacteriana e solicitada broncoscopia para melhor investigação. A pesquisa de fungos no lavado broncoalveolar (LBA) revelou numerosas hifas com raros septos 


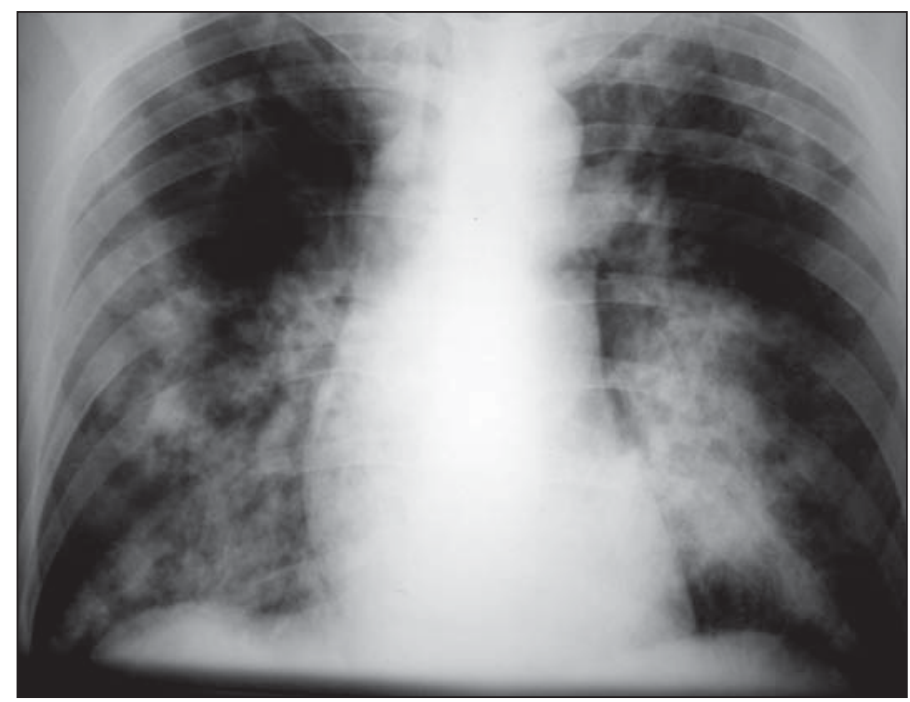

Figura 1. Radiografia simples de tórax mostra áreas de hipotransparência nodular e broncogramas aéreos bilateralmente. bifurcados sugestivos de Aspergillus sp. (Figura 3). A cultura do LBA foi positiva para Nocardia sp. (Figura 4) e, posteriormente, para Mycobacterium tuberculosis (Figura 5). Foi suspensa a antibioticoterapia e foram instituídas terapias com anfotericina B, sulfametoxazol-trimetoprim e anti-retrovirais.

Durante a internação o paciente apresentou quadro diarréico, tendo sido diagnosticada criptosporidiose intestinal. Após 20 dias, recebeu alta hospitalar sem queixas pulmonares e com resolução da diarréia. Decorridos 15 dias, retornou apresentando diarréia, febre, disfagia e emagrecimento importante. Após cinco dias, o paciente foi a óbito por sepse estafilocócica com origem em foco pulmonar.

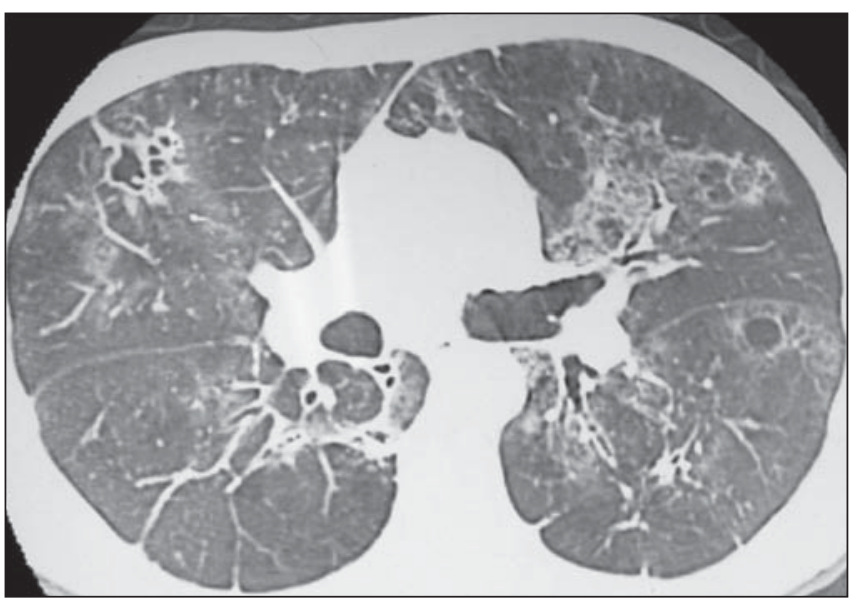

A

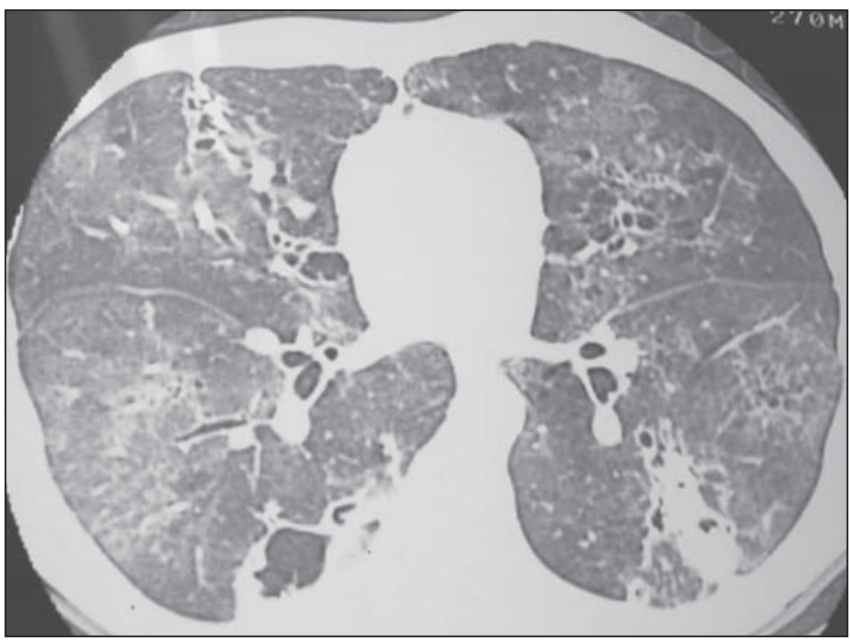

C

Figura 2. Tomografia computadorizada do tórax mostra áreas de consolidação, cavitação, bronquiectasia, opacidade em vidro fosco, espessamento intersticial e broncogramas aéreos, distribuídos difusa e bilateralmente.

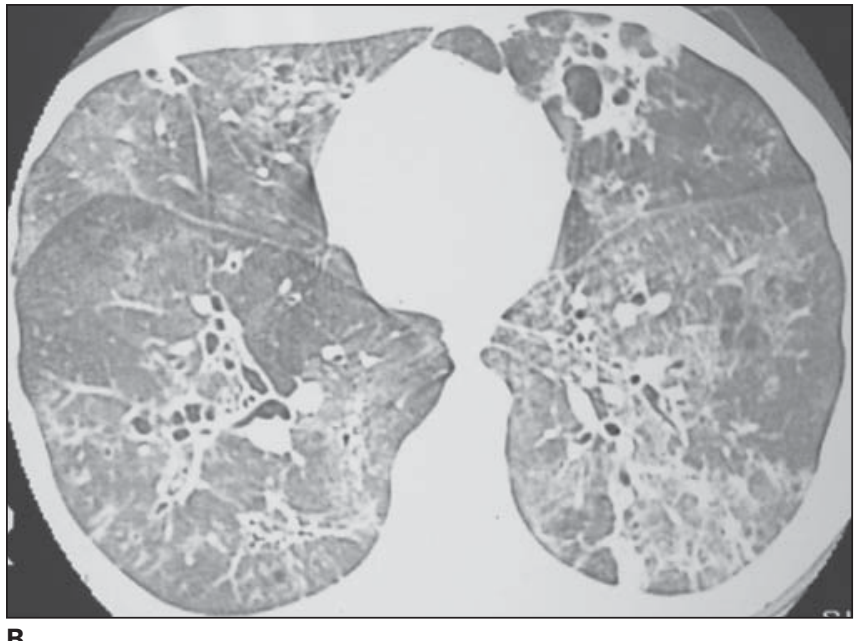

B

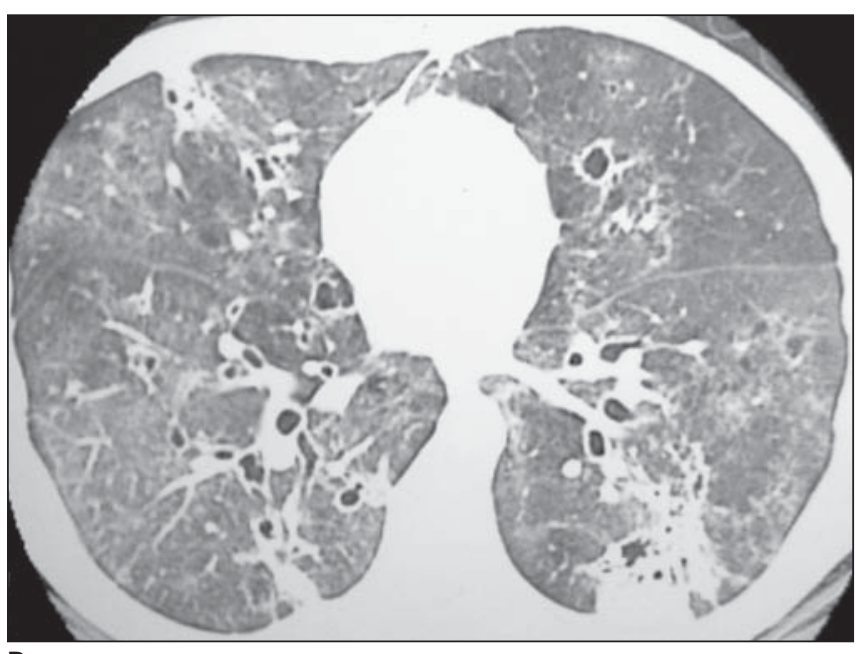

D 


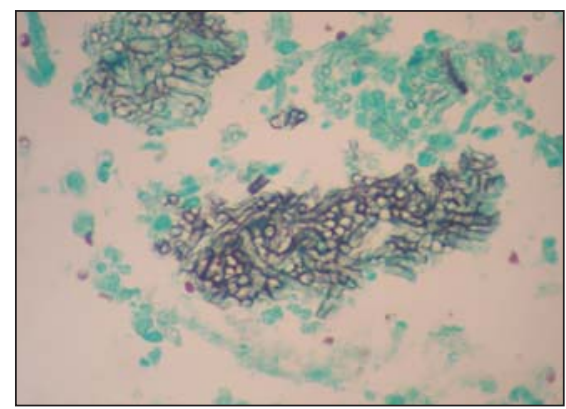

Figura 3. Microscopia com coloração de Grocott demonstra hifas hialinas septadas ramificadas dicotomicamente em ângulo de $45^{\circ}$ (Aspergillus sp.).

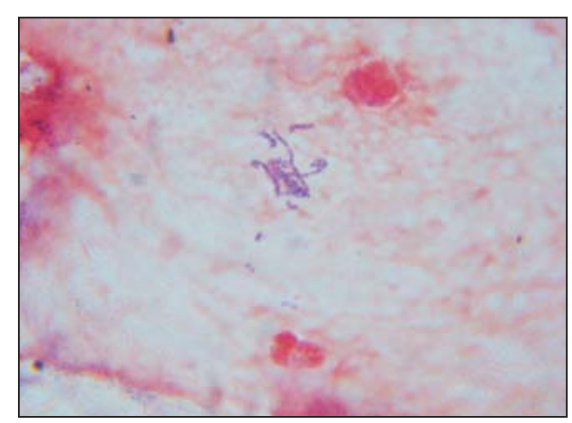

Figura 4. Microscopia evidencia bactéria filamentosa, Gram-positiva, álcool-ácido resistente, característico da Nocardia sp.

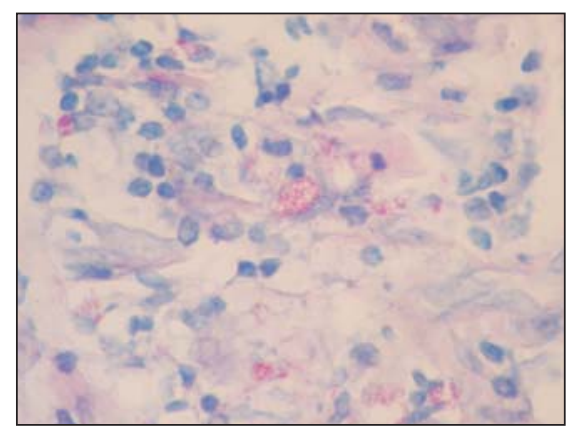

Figura 5. Microscopia com coloração de Ziehl-Nielsen mostrando bacilo álcool-ácido resistente (Mycobacterium tuberculosis).

\section{DISCUSSÃO}

\section{Tuberculose}

A infecção por Mycobacterium tuberculosis inicia-se quando gotículas contendo o organismo vivo são inaladas por pessoas suscetíveis a terem a doença. Quando alcançam o pulmão, os organismos são ingeridos por macrófagos e, ou morrem, ou persistem e multiplicam-se.

Pessoas infectadas pelo HIV e com SIDA têm risco aumentado de cerca de 500 vezes em desenvolver tuberculose ${ }^{(\mathbf{1})}$.
Os métodos diagnósticos de "screening" em pacientes com SIDA e tuberculose pulmonar ativa são pouco eficientes e muito discutidos atualmente. A eficácia do teste da tuberculina é de $50-70 \%$ e o da detecção de BAAR através do exame de escarro e do LBA é menor que 50\% nesses pacientes ${ }^{(2-4)}$. Por isso, foi recomendado pelo Centro de Controle e Prevenção de Doenças dos Estados Unidos que todos os pacientes soropositivos e seus contatos fizessem "screening" com radiografia de tó$\operatorname{rax}^{(2,5,6)}$. Porém, Greenberg et al. ${ }^{(7)}$ analisaram os diferentes achados radiológicos pulmonares em 133 pacientes com SIDA e tuberculose pulmonar ativa e viram que, destes, 38 (28\%) tinham o padrão pós-primário típico e 48 (36\%), o padrão primário típico - 25 (19\%) com linfonodomegalia torácica, $23(17 \%)$ com opacificação lobar e $13(10 \%)$ com derrame pleural. Viram ainda que 19 (14\%) pacientes apresentavam radiografia simples de tórax normal, $17(13 \%)$ mostraram opacificação atípica, $4(3 \%)$ apresentaram padrão miliar e $7(5 \%)$ tinham mínimas alterações radiológicas, mostrando as variadas formas de apresentação radiológica da tuberculose pulmonar ativa em pacientes com SIDA. Assim, Greenberg et al. ${ }^{(7)}$ concluíram que os achados radiológicos são inespecíficos e que para aumentar a eficácia no diagnóstico há a necessidade de se fazer análise combinada dos métodos de "screening" (tanto exames laboratoriais como radiológico) associada à história clínica.

O tratamento da tuberculose pulmonar em pacientes imunodeprimidos e com peso superior a $45 \mathrm{~kg}$ é realizado com rifampicina $600 \mathrm{mg} /$ dia durante seis meses, isoniazida $400 \mathrm{mg} /$ dia também por seis meses e pirazinamida $2.000 \mathrm{mg} /$ dia durante dois meses.

\section{Nocardiose}

Nocardia sp. é uma bactéria aeróbica filamentosa presente no solo e responsável pela nocardiose pulmonar ou sistêmica ${ }^{(8)}$. A Nocardia asteroides é responsável por cerca de $80 \%$ da nocardiose pulmonar ${ }^{(9)}$. Já a Nocardia brasiliensis, em geral, causa infecções cutâneas e subcutâneas localizadas ${ }^{(10)}$ e, menos comumente, também afetam o pulmão produzindo lesões idênticas às da Nocardia asteroides ${ }^{(\mathbf{1 1})}$.
A nocardiose pulmonar tipicamente ocorre em pacientes imunocomprometidos, especialmente os tratados com drogas antiinflamatórias, particularmente corticosteróides, para doença pulmonar obstrutiva crônica e outras doenças sistêmicas como colagenoses, sarcoidose, neoplasias e transplante cardíaco ${ }^{(\mathbf{9 , 1 2 , 1 3})}$. A incidência de nocardiose é bastante baixa em pacientes imunocomprometidos pela infecção por HIV. Isto pode ser explicado pela ampla utilização de sulfonamidas, droga de escolha para tratamento da nocardiose, para profilaxia da infecção por Pneumocystis carinii em pacientes com SIDA ${ }^{(\mathbf{1 4})}$.

As principais alterações patológicas produzidas pela Nocardia sp. no pulmão são abscessos necrotizantes e formação de

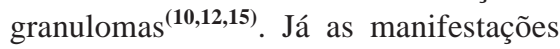
radiológicas são inespecíficas, sendo consolidações e grandes nódulos irregulares com cavitações os achados mais comuns; além destes, nódulos, massas e espessamento intersticial também podem ocorrer $^{(\mathbf{1 2})}$. Derrame pleural e linfonodomegalia são relativamente freqüientes ${ }^{(16,17)}$.

O diagnóstico de nocardiose é bastante difícil, pois sua cultura é demorada (cerca de três semanas), não existem testes sorológicos e as manifestações radiológicas, em geral, são inespecíficas ${ }^{(17)}$.

O tratamento da nocardiose da forma pulmonar é feito com sulfametoxazol 75 $\mathrm{mg} / \mathrm{kg} /$ dia e trimetoprim $15 \mathrm{mg} / \mathrm{kg} /$ dia divididos em duas a quatro doses por dia, durante quatro semanas. Após esse período, a dose de trimetoprim deve ser reduzida para $10 \mathrm{mg} / \mathrm{kg} / \mathrm{dia}$ por um período de seis meses. Tratamento alternativo pode ser feito com imipenem $500 \mathrm{mg}$ intravenoso de seis em seis horas, associado a amicacina $7,5 \mathrm{mg} / \mathrm{kg}$ intravenosa de 12 em 12 horas.

Assim, a nocardiose pulmonar é entidade que sempre deve ser incluída como diagnóstico diferencial nos casos de pacientes imunocomprometidos, particularmente naqueles que fazem uso crônico de corticosteróide e que apresentam achados por imagem sugestivos da doença.

\section{Aspergilose}

Aspergilose é uma doença micótica causada por espécies de Aspergillus, especialmente o Aspergillus fumigatus, que são fungos ubíquos que vivem no solo. 
As manifestações histológicas, clínicas e radiológicas da aspergilose pulmonar são determinadas pelo número e virulência do fungo e pela resposta imune dos hospedei$\operatorname{ros}^{(18-20)}$, sendo, assim, freqüentemente vista em pacientes imunocomprometidos.

A aspergilose pulmonar é subdividida em cinco categorias: a) aspergilose saprofítica (aspergiloma); b) aspergilose broncopulmonar alérgica (reação por hipersensibilidade); c) aspergilose semi-invasiva (necrotizante crônica); d) aspergilose invasiva das vias aéreas; e) aspergilose angioin$\operatorname{vasiva}^{(21)}$.

As manifestações radiológicas são bastante variadas, dependendo do subtipo da doença. A TC de alta resolução (TCAR) é o método por imagem de escolha para avaliação da aspergilose pulmonar. A forma aspergiloma é caracterizada por massa com atenuação de tecidos moles dentro de uma cavidade pulmonar. Essa massa é tipicamente separada da parede da cavidade por um espaço de ar ("sinal do ar-crescente") e está freqüientemente associada com espessamento da parede e pleura adjacentes ${ }^{(21)}$. Os achados radiológicos da aspergilose broncopulmonar alérgica consistem em impactação mucóide e bronquiectasia envolvendo predominantemente os lobos superiores $^{(\mathbf{2 1})}$. A forma necrotizante crônica pode manifestar-se como massa endobrônquica, pneumonite obstrutiva ou massa hilar ${ }^{(21)}$. Bronquiolite (na forma invasiva das vias aéreas) é caracterizada por apresentar-se como nódulos centrolobulares e pelo padrão de "árvore em brotamento"(21). As características tomográficas da aspergilose angioinvasiva consistem de nódulos circundados por halo com atenuação em vidro fosco ("sinal do halo") ou por áreas de consolidação adjacentes à pleura ${ }^{(21)}$.

O tratamento da forma angioinvasiva é feito com anfotericina B, dose de $1 \mathrm{mg} / \mathrm{kg}$ / dia intravenoso, até dose total de 2-2,5 g. Já o tratamento da forma necrotizante é realizado com itraconazol $200 \mathrm{mg} / \mathrm{dia} \mathrm{du}-$ rante três meses, além de intervenção cirúrgica para retirada da lesão.

Assim, a aspergilose pulmonar é complicação séria que frequientemente é vista em pacientes imunocomprometidos. Apesar dos achados por imagem dos vários tipos de aspergilose pulmonar serem inespecíficos, numa colocação clínica adequada e familiaridade com os achados da TCAR, pode-se sugerir ou mesmo ajudar a estabelecer o diagnóstico específico.

\section{CONCLUSÃO}

Este caso chama grande atenção devido à tripla infecção pulmonar (tuberculose, nocardiose e aspergilose) em paciente gravemente imunodeprimido pelo HIV. Apesar de em pacientes com SIDA existir a possibilidade de ocorrer múltiplos focos infecciosos, não encontramos descrições na literatura de acometimento triplo do sistema respiratório em pacientes imunocomprometidos. Assim, concluímos que, mesmo que remota, existe esta possibilidade e devemos sempre acrescentá-la no diagnóstico diferencial.

\section{REFERÊNCIAS}

1. Barnes PF, Bloch AB, Davidson PT, Snider DE Jr. Tuberculosis in patients with human immunodeficiency virus infection. N Engl J Med 1991;324: 1644-1650.

2. Fitzgerald JM, Grzybowski S, Allen EA. The impact of human immunodeficiency virus infection on tuberculosis and its control. Chest 1991;100:191200.

3. Klein NC, Duncanson FP, Lenox TH III, Pitta A, Cohen SC, Wormser GP. Use of mycobacterial smears in the diagnosis of pulmonary tuberculosis in AIDS/ARC patients. Chest 1989;95:1190-1192.

4. Kramer F, Modilevski T, Waliany AR, Leedom JM, Barnes PF. Delayed diagnosis of tuberculosis in patients with human immunodeficiency virus infection. Am J Med 1990;89:451-456.

5. Centers for Disease Control. Screening for tuber- culosis and tuberculous infection in high-risk populations and the use of preventive therapy for tuberculous infection in United States: Recommendations of the Advisory Committee for the Elimination of Tuberculosis. MMWR 1990;39:1-12.

6. Barnes PF, Verdegem TD, Vachon LA, Leedom JM, Overturf GD. Chest roentgenogram in pulmonary tuberculosis. New data on an old test. Chest 1988; 94:316-320

7. Greenberg SD, Frager D, Suster B, Walker S, Stavropoulos C, Rothpearl A. Active pulmonary tuberculosis in patients with AIDS: spectrum of radiographic findings (including a normal appearance) Radiology 1994;193:115-119.

8. Tierney LM Jr, McPhee SJ, Papadakis MA. Nocardiosis. In: Chambers HF, editor. Current medical diagnosis and treatment. 38th ed. Stamford, Conn: Appleton \& Lange, 1999;1324.

9. Beaman BL, Burnside J, Edwards B, Causey W. Nocardial infections in the United States, 19721974. J Infect Dis 1976;134:286-289.

10. Rubin E, Shin MS. Pleural and extrapleural disease in Nocardia infections. J Can Assoc Radiol 1984; 35:189-191

11. Smego RA Jr, Gallis HA. The clinical spectrum of Nocardia brasiliensis infection in the United States. Rev Infect Dis 1984;6:164-180.

12. Murray JF, Finegold SM, Froman S, Will DW. The changing spectrum of nocardiosis. A review and presentation of nine cases. Am Rev Respir Dis 1961;83:315-330.

13. Krick JA, Stinson EB, Remington JS. Nocardia infection in heart transplant patients. Ann Intern Med 1975;82:18-26.

14. Roca B, Cabrera E, Albert M, Chamocho MI. Nocardiosis e infección por el VIH. Rev Clin Esp 2004;204:237-238.

15. Presant CA, Wiernik PH, Serpick AA. Factors affecting survival in nocardiosis. Am Rev Respir Dis 1973;108:1444-1448

16. Balikian JP, Herman PG, Kopit S. Pulmonary nocardiosis. Radiology 1978;126:569-573.

17. Feigin DS. Nocardiosis of the lung: chest radiographic findings in 21 cases. Radiology 1986;159: 9-14.

18. Greene R. The pulmonary aspergilloses: three distinct entities or a spectrum of disease. Radiology 1981;140:527-530.

19. Klein DL, Gamsu G. Thoracic manifestations of aspergillosis. AJR 1980;134:543-552.

20. Gefter WB. The spectrum of pulmonary aspergillosis. J Thorac Imaging 1992;7:56-74.

21. Franquet T, Müller NL, Giménez A, Guembe P, de la Torre J, Bagué S. Spectrum of pulmonary aspergillosis: histologic, clinical, and radiologic findings. RadioGraphics 2001;21:825-837. 